\title{
It is not all about BRCA: Cullin-Ring ubiquitin Ligases in ovarian cancer
}

\author{
A Carlucci ${ }^{1,2}$ and V D'Angiolella*,1 \\ ${ }^{1}$ Cancer Research UK and Medical Research Council Oxford Institute for Radiation Oncology, Old Road Campus, Oxford OX3 \\ 7DQ, UK and ${ }^{2}$ Department of Experimental and Clinical Medicine, Magna Græcia University of Catanzaro, Salvatore Venuta \\ Campus, Viale Europa, 88100 Catanzaro, Italy
}

Mutations in the BRCA1 and BRCA2 genes predispose individuals to the development of breast and ovarian cancers. As a result, biochemical functions of BRCA1 and BRCA2 proteins are being characterised in great detail. These studies have prompted the use of PARP inhibitors to treat BRCA1 and BRCA2-deficient ovarian cancers. This example of synthetic lethality represents a conceptual progress made recently in the approach to cancer treatment and is being currently tested in multiple clinical trials. Other than BRCA1 and BRCA2, many other factors might influence the survival of ovarian cancer patients. Currently, ovarian cancer remains the fifth most common cancer in the United Kingdom among women. Recent evidence suggests benefit in the modulation of the ubiquitin-proteasome system for the treatment of ovarian cancer. In this manuscript, we review the role of Cullin-Ring ubiquitin Ligases (CRLs) in the pathogenesis of ovarian cancer and their potential therapeutic exploitation. CRLs comprise a large family of proteins that, like kinases, might represent ideal candidates for targeted therapy and provide a large repertoire for the development of new anti-cancer compounds.

The growth and function of every single cell within tissues is carefully coordinated with resources evenly distributed and efficiently utilised. Cells proliferate to allow growth, facilitate wound repair and replace non-functional cells. Like in every complex system, there is a certain level of stochasticity and uncontrolled cell proliferation can take over. Loss of control in the cells of ovarian tissues can lead to the development of ovarian cancer, which is the fifth most common cancer among women in the United Kingdom (http://www.ons.gov.uk/).

The ovary is composed of three diverse cellular compartments and tumours can arise from uncontrolled proliferation in each one of them. Accordingly, ovarian cancers can be classified in epithelial carcinomas, germ cell tumours and sex-cord stromal tumours. These cancers are genetically different and have different prognosis and incidence. For instance, the most commonly diagnosed ovarian cancer is the epithelial carcinoma, which includes serous tumours, endometrioid tumours, mucinous cystadenocarcinomas, Brenner tumours, and transitional cell carcinomas of the ovary. Germ cell tumours and sex-cord stromal tumours tend to be more benign and are less frequently observed.

Large sequencing studies have unveiled the major genetic aberrations in epithelial cancers. Hence, in the future, we will be able to tailor cancer therapy according to the genetic alterations within the tumour. At the moment, despite being able to identify the basic composition of many tumour genomes, epithelial ovarian cancers are diagnosed late due to their anatomical location and lack of cancer-specific symptoms. At the diagnosis stage, metastases of the primary ovarian tumour are beyond its originating tissue and the prognosis is often poor, with a $42.9 \% 5$-year survival rate (independently of disease stage) (http://www.ons.gov.uk/). The current standard therapy is the surgical resection of the tumour, followed by paclitaxel in combination with platinum-based chemotherapy treatment.

The genome of ovarian cancers contains aberrations in the components of the ubiquitin-proteasome system, which mediates the controlled degradation of targeted proteins. The destiny of proteins is dictated by a signalling pathway that creates a 'degron'(Figure 1), a docking site for E3 ubiquitin ligases to add chains of ubiquitin monomers. An activated ubiquitin molecule is transferred from a ubiquitin-activating enzyme E1 to a ubiquitin-conjugating enzyme E2. The activated ubiquitin on the E2 is then moved to target substrates through the use of E3s that recognise 'degrons' for substrate specificity. The addition of ubiquitin molecules to target proteins induces the formation of chains that are recognised by the proteasome for substrate degradation (Figure 1).

*Correspondence: Dr V D’Angiolella; E-mail: vincenzo.dangiolella@oncology.ox.ac.uk

Received 23 May 2014; revised 2 October 2014; accepted 9 October 2014;

published online 9 December 2014

(c) 2015 Cancer Research UK. All rights reserved 0007-0920/15

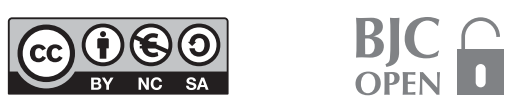




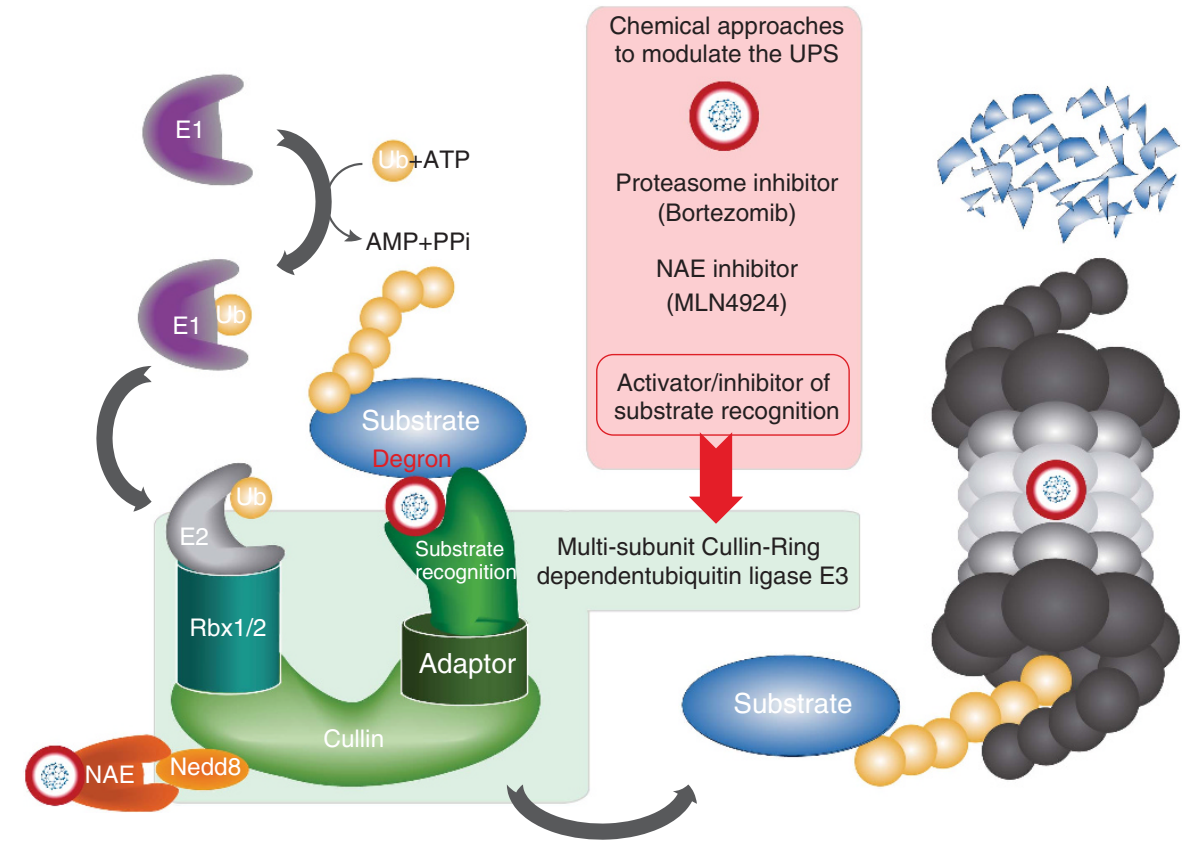

Figure 1. Cullin-Ring ubiquitin Ligases (CRLs) are prototypical multi-subunit ubiquitin ligases. This figure presents the assembly of a prototypical multi-subunit E3 through adaptors. Substrates contain a degron that serves as a signalling platform for the recruitment by the E3. Rbx1/2 are recruited by cullins to bridge the substrate selected by the substrate recognition protein to the E2. E2 and E1 provide activated ubiquitin molecules that are coupled with target lysine residues within the substrates. Poly-ubiquitinated substrates are degraded by the proteasome. NAE (Nedd8-activating enzyme) catalyses the transfer of Nedd8 to cullins. Chemical approaches to target the CRLs are outlined.

BRCA1 and BRCA2 are susceptibility loci that predispose individuals to the development of ovarian and breast cancers. The protein product of $B R C A 1$ gene codes for an E3 ubiquitin ligase and BRCA2 acts as a scaffold to favour Rad51 recruitment at single strand DNA. BRCA1 and BRCA2 are both required for the repair of double strand break (DSB) lesions within DNA (Aparicio et al, 2014). Specifically, these proteins allow the correct execution of Homologous Recombination (HR) in mammalian cells for the efficient repair of DSBs. The loss of BRCA1 and BRCA2 represents a pathogenetic mechanism as it favours genome instability, but it also points to a specific cancer weakness. Indeed, by inducing DSBs in cancer cells that lack BRCA function, it is possible to sensitise specifically these cells and leave normal tissue intact, given the inability of these cancer cells to properly handle DSBs. It was shown that PARP inhibitors sensitise cancer cells-bearing BRCA1 and BRCA2 mutations (Bryant et al, 2005), through the obligate use of BRCA1/2 for repair of DSBs. This evidence is among the first applied examples of synthetic lethality in cancer therapy. Ovarian cancer patients might benefit from the treatment with PARP inhibitors and numerous clinical trials attempting to confirm these benefits are in progress.

Although several factors besides BRCA1/2 influence success of therapy and cancer cell survival, these might be masked by the profound genetic heterogeneity of cancers. In the following review, we discuss the role of Cullin-Ring ubiquitin Ligases (CRLs) in the pathogenesis of epithelial ovarian cancer and their potential therapeutic exploitation.

\section{CULLIN-RING UBIQUITIN LIGASES ORGANISATION AND CHEMICAL TARGETING}

The ubiquitin system is typically organised in multiple layers of specificity. The human genome codes for two E1s, $\sim 35 \mathrm{E} 2 \mathrm{~s}$ and more than 500 E3 ubiquitin ligases. Cullin proteins assemble the largest class of RING E3 ligases known as CRLs. Nine cullins have been described and Cul1, Cul2, Cul3, Cul4, Cul5, Cul7, Cul9 have
Table1. Organisation of Cullin-Ring ubiquitin Ligases (CRLs)

\begin{tabular}{|l|l|l|l|l|}
\hline $\begin{array}{l}\text { Cullin-RING } \\
\text { E3 ligases }\end{array}$ & RING & Protein & Adaptor & $\begin{array}{l}\text { Substrate } \\
\text { recognition }\end{array}$ \\
\hline CRL1/SCF & ROC1/Rbx1 & CUL1 & Skp1 & F-box protein \\
\hline CRL2 & ROC1/Rbx1 & CUL2 & EloC/EloB & VHL box \\
\hline CRL3 & ROC1/Rbx1 & CUL3 & BTB & BTB \\
\hline CRL4 (A/B) & ROC1/Rbx1 & CUL4 & DDB1 & DCAF \\
\hline CRL5 & ROC2/Rbx2 & CUL5 & EloC/EloB & SOCS protein \\
\hline CRL7 & ROC1/Rbx1 & CUL7 & Skp1 & Fbw8 \\
\hline CRL9 & ROC1/Rbx1 & PARC & - & - \\
\hline
\end{tabular}

been shown to assemble with a diverse family of E3s (Table 1). The general feature is that cullins bridge together with a ring finger protein $(\mathrm{RBX} 1 / 2)$ and an adaptor (Skp1, ElonginC/B, DDB1). The ring finger protein $(\mathrm{RBX} 1 / 2)$ recruits the $\mathrm{E} 2$ whereas the adaptor recruits the E3. An activated ubiquitin molecule is transferred from the E2 to the substrates through substrate recruitment mediated by the E3. The system allows for the regulation of protein ubiquitination in space and time with high selectivity due to the large number of substrate recognition domains present in the E3s with unique features. A further layer of CRLs control is mediated by the Nedd8, a ubiquitin like molecule that is added to the cullins in order to promote the ubiquitinating activity of the CRLs complexes (Figure 1). Neddylation of cullins is catalysed in a manner similar to ubiquitination for which the molecular details have been analysed in a recent elegant study (Scott et al, 2014).

Modulation of the ubiquitin system is exploited in a number of cancer therapies. Bortezomib (Velcade, http://www.velcade.com/ files/PDFs/VELCADE_PRESCRIBING_INFORMATION.pdf), a general inhibitor of the proteasome (Adams and Kauffman, 2004), is used in the treatment of patients with multiple myeloma and mantle cell lymphoma. Novel drugs that target the ubiquitination system have been developed and are currently being tested in clinical trials. For instance, MLN4924, a Nedd8-activating 
enzyme inhibitor, prevents the neddylation of cullins and hampers the activity of all the CRLs (Soucy et al, 2009). MLN4924 is being tested in the treatment of haematological malignancies and solid tumours (www.clinicaltrials.gov). In vitro, it was shown that MLN4924 possesses significant cytotoxic activity against both cisplatin-sensitive and -resistant ovarian cancer cells (Nawrocki et al, 2013). These data suggest that CRLs might represent important targets in ovarian cancer therapy; however, more studies are required to define the contribution of each E3 to the observed effect. Although it was shown that Cul3-dependent ubiquitin ligases mediate the outcome of MLN4924 in ovarian cancer cell lines, it was also suggested that Cull siRNA has an opposite effect and increased cisplatin resistance (Jazaeri et al, 2013). Furthermore, recent data showed that Cul4a could mediate the effect of MLN4924 as it was observed that siRNA targeting the E3 ubiquitin ligase DCAF2/CTD2 induces drug sensitivity similar to that observed with MLN4924 treatment (Pan et al, 2013). The emerging picture is not clear, but it indicates that a better definition of the biological role of E3 ubiquitin ligases might lead to significant improvements in chemotherapy regimens, as well as the development of new targeted compounds in ovarian cancer. It is worth mentioning that recent evidence suggests that lenalidomide (Revlimid, http://www.revlimid.com/wp-content/uploads/2013/11/ PI.pdf), currently used in the treatment of multiple myelomas, myelodysplastic syndrome and mantle cell lymphomas, exerts its anti-cancer activity by promoting the recognition of IKFZ1 and IKZF3 by the CRL4, Cereblon (Lu et al, 2014). The discovery that lenalidomide can act as a decoy for E3 ubiquitin ligases opens the possibility to modulate the E3 activity, revealing novel therapeutic routes. Unfortunately, at this moment, the function of many E3 enzymes is poorly characterised and molecular mechanisms of action remain unknown for most of them.

\section{CUL1-DEPENDENT UBIQUITIN LIGASES}

Prototypical multi-subunit ubiquitin ligases are the Cul1-dependent E3s. Through Skp1, they recruit the F-box proteins for substrate recognition. $69 \mathrm{~F}-$ box proteins are present in mammalian cells with multiple roles in cell signalling, proliferation and development. F-box proteins differ notably in their substrate recruitment strategy and function. They are also classified as Fbxw's, Fbxl's and Fbxo's. The Fbxw's contain an F-box domain and a WD-40 motif that mediates the interaction with target substrates through specific serine and threonine phosphorylation consensus sequences. Fbxl's contain leucine-rich repeats and also seem to prefer phosphorylated substrates, while the rest of the F-box protein family (FBXs) lack either WD-40 repeats or leucinerich repeats (Jin et al, 2004).

The Fbxos have a wide range of protein recognition domains, such as a helicase domain in Fbx18, a cyclin domain in Fbxo1 (cyclin F) and many others. For a comprehensive classification, please refer to Jin et al (2004).

The Fbxw7 is among the most well-characterised Fbxw's and its role in cancer has been extensively studied. Fbxw7 is a tumour suppressor that is frequently mutated in multiple cancer types. Fbxw7 targets the products of several oncogenes for degradation, including cyclin E, NOTCH, JUN and c-Myc (Minella and Clurman, 2005). Surprisingly enough, mutations in Fbxw7 are not common in serous ovarian cancers. We have analysed the essentiality of each F-box in ovarian cancer cell lines using the DSPC database (http://dpsc.ccbr.utoronto.ca/cancer/), which is a collection of shRNA dropout signature profiles, covering approximately 16000 human genes derived from more than 70 pancreatic, ovarian and breast cancer human cell lines. We have plotted each F-box according to its cumulative GARP score (derived from the sum of GARP score in individual ovarian cell lines). The GARP score quantifies the shRNA dropout rate of a gene, based on the GARP (Gene Activity Ranking Profile). According to the database, Fbxw7 is required for the survival of the following ovarian cancer cell lines (GARP score is indicated in parenthesis): OVCA1369_TR ( - 2.89); OVCA8 $(-0.54)$; SK-OV3 $(-0.28)$; A2780 $(-0.28)$. The latter indicates that Fbxw7 could also act as an oncogene in certain instances and indeed, it was recently reported that Fbxw7 targets p100, a component of the NF$\kappa \mathrm{B}$ pathway, for degradation as a pro-survival mechanism in multiple myeloma (Busino et al, 2012). Although in other cancers cyclin $\mathrm{E}$ amplification is mediated by Fbxw7 loss, in ovarian cancer, cyclin $\mathrm{E}$ is amplified through locus amplification. Hence, it will be interesting to analyse in more detail the survival requirement of Fbxw7 in ovarian cancer subtypes.

In our analysis, we have plotted the F-boxes with low GARP value (lower GARP value indicates higher growth control) with the total number of mutations in ovarian cancer, derived from the Catalogue Of Somatic Mutations In Cancer (COSMIC) database. Although simplistic, this approach correlates the function of a protein to the presence of mutations (Figure 2). The presence of mutations might not always predict high significance of a certain gene. Poorly transcribed regions in the ovarian cancer genome might be more mutated simply because transcription-coupled repair is not active in these regions. However, if a particular gene is required for cell growth and this requirement is coupled to the presence of mutations in the gene, this could reflect a functional significance even in the presence of a low mutation rate.

In a recent genome-wide sequencing of ovarian cancers, mutational signatures were clustered in pathways (Cancer genome atlas research network, 2011). According to this analysis, alteration of $\mathrm{HR}$ repair is a frequent event. In fact, it has been observed that $20 \%$ of high grade serous ovarian cancer (HGS-OvCa) samples contain somatic and germline mutations in BRCA $1 / 2$ and $11 \%$ have lost BRCA1 expression through DNA hypermethylation. Mutations in other components of $\mathrm{HR}$ and

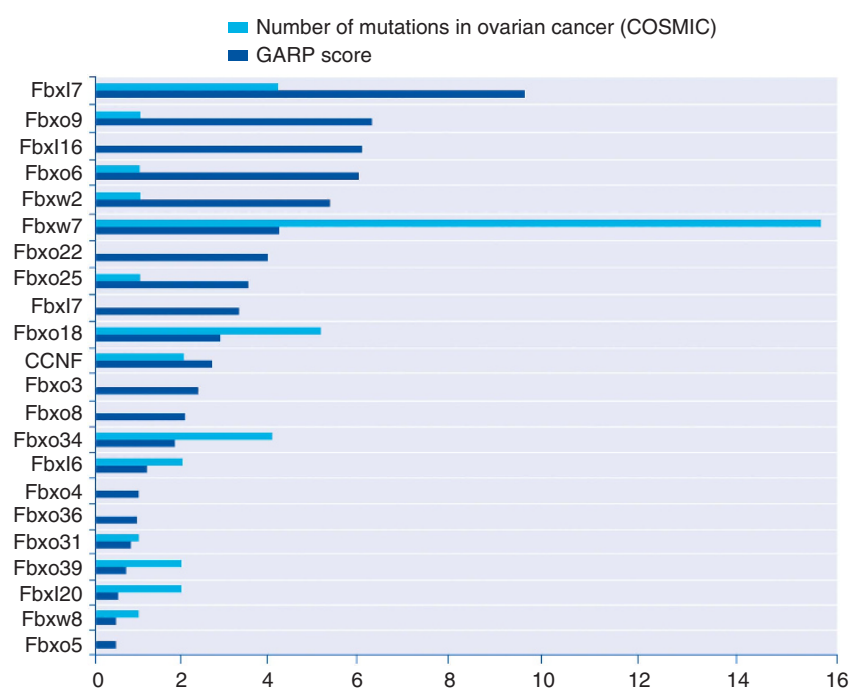

Figure 2. Analysis of the cumulative GARP score derived from the DPSC-cancer database and number of mutations in ovarian cancer from COSMIC of the indicated F-box proteins. GARP scores are reported as positive values, however GARP score is derived as a negative score. For details, please refer to (http://dpsc.ccbr.utoronto. $\mathrm{ca} /$ cancer/). Number of mutations identified in the COSMIC database (ovarian cancer subset) (http://cancer.sanger.ac.uk/cancergenome/ projects/cosmic/)were counted for the F-box proteins scoring in the DPSC database. Please note synonymous mutations have been omitted. F-box proteins not included in the graph did not score in the DPSC. 
DNA damage response (ATM/ATR, components of the Fanconi anaemia core complex and Rad51 mutations) can also take place. Considering the summation of all the DNA damage response genes altered, it has been calculated that HR defects might be present in approximately half of all HGS-OvCa cases (Cancer genome atlas research network, 2011). This observation could have a major therapeutic relevance as cancers with HR defects can benefit from PARP inhibitors-based therapy. Our analysis suggests that Fbxo18 has a low GARP score in ovarian cancer cell lines and is mutated with low frequency. Interestingly, it was shown that Fbxo18 modulates HR by disrupting Rad51 recruitment (Simandlova et al, 2013). On the light of the role of Fbxo18 in HR, we speculate that the mutations reported in Fbxo18 might be relevant for the treatment of ovarian cancer and could modulate the response to PARP inhibitors. Mutations in Fbxo18 were reported in melanoma, where inactivation of Fbxo18 was shown to protect cells from UV-induced DNA damage (Jeong et al, 2013). Noteworthy, another Fbox - Fbxo44 - has a sugar recognition domain and it was observed that Fbxo44 targets BRCA1 for degradation. Although these findings are interesting, functional significance of the proteolysis of BRCA1 is not yet clear, and it remains unexplained how Fbxo44 mediates BRCA1 for ubiquitination and subsequent degradation through the sugar recognition domain (Lu et al, 2012). In the future, the role of Fbxo18 and Fbxo44 in ovarian cancer will need to be more thoroughly investigated.

Alteration of cell cycle is another common feature in HGSOvCa. For instance, amplification of cyclin D1 and D2 is present in 5 and $15 \%$ of HGS-OvCa, respectively. Fbxo31 was shown to target cyclin D1 for degradation and in our analysis, Fbxo31 is among the F-boxes harbouring mutations and showing essentiality in one cell line (Santra et al, 2009).

Other cell cycle components significantly altered in HGs-OvCa are RB1, p21, p57 and cyclin E. As RB1 can affect the levels of cyclin F (Fbxo1) (D'Angiolella et al, unpublished observation), we hypothesise that alteration of cyclin F levels might be relevant in ovarian cancer progression and prognosis. We have previously shown that cyclin F targets RRM2, a subunit of ribonucleotide reductase, for degradation during the G2 phase of the cell cycle (D'Angiolella et al, 2012, 2013). A statistically significant direct association of RRM2 expression levels and the relative risk of death was observed in serous ovarian cancer (Wang et al, 2012). Moreover, cyclin F is the only F-box together with Fbxl18 that clusters in the survival signature derived from the TCGA analysis of HGS-OvCa (Cancer genome atlas research network, 2011). In light of these observations, it will be interesting to investigate in more detail the roles that cyclin F and RRM2 have in the prognosis and survival of ovarian cancer patients.

Skp2, also known as Fbxl1, acts as an oncogene by targeting p27 for degradation. Regulation of p27 allows cell cycle progression by modulating CDK activity (Carrano et al, 1999). Numerous human cancers, including ovarian cancers, express low levels of p27 and high levels of Skp2. A study showed that Skp2-/- mice have specific developmental defects in the ovary (Fotovati et al, 2011). Thus, blocking Skp2 activity might be beneficial for cancer therapy, especially for ovarian cancer. As a matter of fact, various chemical inhibitors of Skp2 have been developed (Wu et al, 2012; Chan et al, 2013). However, at this moment, the high $\mathrm{IC}_{50}$ (half maximal inhibitory concentration) of these compounds and poor pharmacological properties significantly complicate their development for use in the clinic.

Other F-box proteins that have a described role in the proliferation of ovarian cancer cells are Fbxo22 and Fbxo32. Fbxo22 is among the hits of the 106 cell-line screening performed at the Broad Institute. siRNA targeting of Fbxo22 can block specifically the growth of ovarian cancer cell lines (Cheung et al, 2011). Fbxo22 targets KDM4A for degradation and alteration of Fbxo22 leads to changes in $\mathrm{H} 3 \mathrm{~K} 9 \mathrm{me} 3$ and $\mathrm{H} 3 \mathrm{~K} 36 \mathrm{me} 3$ histone marks, as well as changes in transcriptional levels of KDM4A's target gene, ASCL2 (Tan et al, 2011). The investigation of Fbxo22 in ovarian cancer might reveal novel aspects of epigenetic changes associated with cancer progression. Fbxo32, also known as atrogin, was initially described as an inductor of atrophy, a physiological condition that leads to a reduction in muscle cell size and increased protein catabolism. Fbxo32 is transcriptionally silenced in ovarian cancers and re-expression of Fbxo32 inhibits growth progression of ovarian cancer cells through promotion of apoptosis (Chou et al, 2010). Other F-box proteins included in our analysis might have relevant roles as well, but their functional roles in ovarian cancer cell lines remain unexplored.

\section{INSIGHTS INTO OTHER CRLS}

Among the numerous CRLs, Cull-dependent machineries are the most thoroughly studied. Although other CRLs might also be relevant in the survival of ovarian cancer cells as described above, it is difficult to establish the relative contribution of each one of them as many of these enzymes are poorly characterised at this moment.

Cullin-Ring ubiquitin Ligases 4 (CRL4s) use the protein DDB1 as an adaptor and recruit substrates via DDB4 and Cul4 associated factors (DCAFs). The function of the majority of DCAFs is unknown, but recent work has demonstrated that the expression of the E3 DCAF2/CDT2 could serve as a biomarker of ovarian cancer prognosis. Inhibition of CDT2 arrests ovarian cell proliferation and might mediate the effect of MLN4924 on overcoming cisplatin resistance (Birner et al, 2012; Pan et al, 2013).

CRL3s assemble complexes with BTB proteins $(\sim 200$ in the human genome). Inhibition of Cul3 sensitises ovarian cancer cells to cisplatin. Investigation of BTBs in ovarian cancer might reveal the specific BTB mediating cisplatin sensitivity and provide a novel target for combined therapy. It is important to mention that disruption of the Cul3/Keap/Rbxl has been reported in serous ovarian cancer (Martinez et al, 2014), but the significance for platinum-based therapies is unclear.

Given the broad spectrum of the topic, the description of each CRL is outside the scope of this review. Nevertheless, it is easy to envision that given the complexity of CRLs, we will see a large number of discoveries flourish on this interesting class of molecular machineries in the future. We hope that these molecular details will be translated into more effective treatments through the development of compounds that are more specific for the E3 ligases.

\section{CONCLUSION}

Exome sequencing of cancers has drastically improved our understanding of the aetiology and pathogenesis of various cancer types. Unfortunately, the low number of cases, high cytogenetic variability and tumour population heterogeneity, as well as the contamination of samples with stromal components, might render this analysis difficult to interpret and highly variable across studies. Also, recent evidence has suggested that alterations contained in the non-coding regions of the genome and silent mutations might significantly contribute to cancer development (Supek et al, 2014). Thus, although the major cancer drivers can be easily seen in current studies, more functional studies will be necessary to define novel drivers and therapeutical targets. In order to exploit the synthetic lethality principle effectively and develop novel targeted therapies, we will need to aim node components of signalling pathways and increase our understanding about the physiological processes that control cell survival. In addition, we will need to combine several drugs and target multiple pathways to prevent aberrant circuits from developing. Indeed, cancer cells overcome 
easily the targeting of a single genetic aberration through development of resistance. An accumulating body of evidence suggests strongly that multiple genetic aberrations work independently or together to confer survival ability to cancer cells. In summary, it is only the combination of functional studies and genomic signatures that will allow us to make progress in ovarian cancer treatment. In the future, we will need to learn how diverse pathways interact and integrate them in order to predict the development of drug resistance by cancer cells.

\section{ACKNOWLEDGEMENTS}

We would like to thank the the HEMMAS project of Magna Graecia University of Catanzaro, the Union for International Cancer Control (UICC) and Medical Research Council (MRC) UK (MC_PC_12007) for their support. We would like to thank Kai-li Braunbeck and Professor Michele Pagano for critical reading of the manuscript. We would like to thank Bruno Vaz for the preparation of Figure 1.

\section{REFERENCES}

Adams J, Kauffman M (2004) Development of the proteasome inhibitor Velcade (Bortezomib). Cancer Invest 22: 304-311.

Aparicio T, Baer R, Gautier J (2014) DNA double-strand break repair pathway choice and cancer. DNA Repair (Amst) 19: 169-175.

Birner P, Schoppmann A, Schindl M, Dinhof C, Jesch B, Berghoff AS, Schoppmann SF (2012) Human homologue for Caenorhabditis elegans CUL-4 protein overexpression is associated with malignant potential of epithelial ovarian tumours and poor outcome in carcinoma. J Clin Pathol 65: $507-511$.

Bryant HE, Schultz N, Thomas HD, Parker KM, Flower D, Lopez E, Kyle S, Meuth M, Curtin NJ, Helleday T (2005) Specific killing of BRCA2deficient tumours with inhibitors of poly(ADP-ribose) polymerase. Nature 434: 913-917.

Busino L, Millman SE, Scotto L, Kyratsous CA, Basrur V, O'connor O, Hoffmann A, Elenitoba-Johnson KS, Pagano M (2012) Fbxw7alpha- and GSK3-mediated degradation of p100 is a pro-survival mechanism in multiple myeloma. Nat Cell Biol 14: 375-385.

Cancer genome atlas research network (2011) Integrated genomic analyses of ovarian carcinoma. Nature 474: 609-615.

Carrano AC, Eytan E, Hershko A, Pagano M (1999) SKP2 is required for ubiquitin-mediated degradation of the CDK inhibitor p27. Nat Cell Biol 1: 193-199.

Chan CH, Morrow JK, Li CF, Gao Y, Jin G, Moten A, Stagg LJ, Ladbury JE, Cai Z, Xu D, Logothetis CJ, Hung MC, Zhang S, Lin HK (2013) Pharmacological inactivation of Skp2 SCF ubiquitin ligase restricts cancer stem cell traits and cancer progression. Cell 154: 556-568.

Cheung HW, Cowley GS, Weir BA, Boehm JS, Rusin S, Scott JA, East A, Ali LD, Lizotte PH, Wong TC, Jiang G, Hsiao J, Mermel CH, Getz G, Barretina J, Gopal S, Tamayo P, Gould J, Tsherniak A, Stransky N, Luo B, Ren Y, Drapkin R, Bhatia SN, Mesirov JP, Garraway LA, Meyerson M, Lander ES, Root DE, Hahn WC (2011) Systematic investigation of genetic vulnerabilities across cancer cell lines reveals lineage-specific dependencies in ovarian cancer. Proc Natl Acad Sci USA 108: 12372-12377.

Chou JL, Su HY, Chen LY, Liao YP, Hartman-Frey C, Lai YH, Yang HW, Deatherage DE, Kuo CT, Huang YW, Yan PS, Hsiao SH, Tai CK, Lin HJ, Davuluri RV, Chao TK, Nephew KP, Huang TH, Lai HC, Chan MW (2010) Promoter hypermethylation of FBXO32, a novel TGF-beta/SMAD4 target gene and tumour suppressor, is associated with poor prognosis in human ovarian cancer. Lab Invest 90: 414-425.

D’Angiolella V, Donato V, Forrester FM, Jeong YT, Pellacani C, Kudo Y, Saraf A, Florens L, Washburn MP, Pagano M (2012) Cyclin F-mediated degradation of ribonucleotide reductase M2 controls genome integrity and DNA repair. Cell 149: 1023-1034.

D’Angiolella V, Esencay M, Pagano M (2013) A cyclin without cyclin-dependent kinases: cyclin F controls genome stability through ubiquitin-mediated proteolysis. Trends Cell Biol 23: 135-140.
Fotovati A, Abu-Ali S, Nakayama K, Nakayama KI (2011) Impaired ovarian development and reduced fertility in female mice deficient in Skp2. J Anat 218: 668-677.

Jazaeri AA, Shibata E, Park J, Bryant JL, Conaway MR, Modesitt SC, Smith PG, Milhollen MA, Berger AJ, Dutta A (2013) Overcoming platinum resistance in preclinical models of ovarian cancer using the neddylation inhibitor MLN4924. Mol Cancer Ther 12: 1958-1967.

Jeong YT, Cermak L, Guijarro MV, Hernando E, Pagano M (2013) FBH1 protects melanocytes from transformation and is deregulated in melanomas. Cell Cycle 12: 1128-1132.

Jin J, Cardozo T, Lovering RC, Elledge SJ, Pagano M, Harper JW (2004) Systematic analysis and nomenclature of mammalian F-box proteins. Genes Dev 18: 2573-2580.

Lu G, Middleton RE, Sun H, Naniong M, Ott CJ, Mitsiades CS, Wong KK, Bradner JE, Kaelin Jr WG (2014) The myeloma drug lenalidomide promotes the cereblon-dependent destruction of Ikaros proteins. Science 343: 305-309.

Lu Y, Li J, Cheng D, Parameswaran B, Zhang S, Jiang Z, Yew PR, Peng J, Ye Q, $\mathrm{Hu}$ Y (2012) The F-box protein FBXO44 mediates BRCA1 ubiquitination and degradation. J Biol Chem 287: 41014-41022.

Martinez VD, Vucic EA, Thu KL, Pikor LA, Hubaux R, Lam WL (2014) Unique pattern of component gene disruption in the NRF2 inhibitor KEAP1/CUL3/RBX1 E3-ubiquitin ligase complex in serous ovarian cancer. Biomed Res Int 2014: 159459.

Minella AC, Clurman BE (2005) Mechanisms of tumour suppression by the SCF(Fbw7). Cell Cycle 4: 1356-1359.

Nawrocki ST, Kelly KR, Smith PG, Espitia CM, Possemato A, Beausoleil SA, Milhollen M, Blakemore S, Thomas M, Berger A, Carew JS (2013) Disrupting protein NEDDylation with MLN4924 is a novel strategy to target cisplatin resistance in ovarian cancer. Clin Cancer Res 19: $3577-3590$.

Pan WW, Zhou JJ, Yu C, Xu Y, Guo LJ, Zhang HY, Zhou D, Song FZ, Fan HY (2013) Ubiquitin E3 ligase CRL4(CDT2/DCAF2) as a potential chemotherapeutic target for ovarian surface epithelial cancer. J Biol Chem 288: 29680-29691.

Santra MK, Wajapeyee N, Green MR (2009) F-box protein FBXO31 mediates cyclin D1 degradation to induce G1 arrest after DNA damage. Nature 459: $722-725$.

Scott DC, Sviderskiy VO, Monda JK, Lydeard JR, Cho SE, Harper JW, Schulman BA (2014) Structure of a RING E3 trapped in action reveals ligation mechanism for the ubiquitin-like protein NEDD8. Cell 157: 1671-1684.

Simandlova J, Zagelbaum J, Payne MJ, Chu WK, Shevelev I, Hanada K, Chatterjee S, Reid DA, Liu Y, Janscak P, Rothenberg E, Hickson ID (2013) FBH1 helicase disrupts RAD51 filaments in vitro and modulates homologous recombination in mammalian cells. J Biol Chem 288: 34168-34180.

Soucy TA, Smith PG, Milhollen MA, Berger AJ, Gavin JM, Adhikari S, Brownell JE, Burke KE, Cardin DP, Critchley S, Cullis CA, Doucette A, Garnsey JJ, Gaulin JL, Gershman RE, Lublinsky AR, McDonald A, Mizutani H, Narayanan U, Olhava EJ, Peluso S, Rezaei M, Sintchak MD, Talreja T, Thomas MP, Traore T, Vyskocil S, Weatherhead GS, Yu J, Zhang J, Dick LR, Claiborne CF, Rolfe M, Bolen JB, Langston SP (2009) An inhibitor of NEDD8-activating enzyme as a new approach to treat cancer. Nature 458: 732-736.

Supek F, Minana B, Valcarcel J, Gabaldon T, Lehner B (2014) Synonymous mutations frequently act as driver mutations in human cancers. Cell 156: 1324-1335.

Tan MK, Lim HJ, Harper JW (2011) SCF(FBXO22) regulates histone H3 lysine 9 and 36 methylation levels by targeting histone demethylase KDM4A for ubiquitin-mediated proteasomal degradation. Mol Cell Biol 31: 3687-3699.

Wang LM, Lu FF, Zhang SY, Yao RY, Xing XM, Wei ZM (2012) Overexpression of catalytic subunit M2 in patients with ovarian cancer. Chin Med J (Engl) 125: 2151-2156.

Wu L, Grigoryan AV, Li Y, Hao B, Pagano M, Cardozo TJ (2012) Specific small molecule inhibitors of Skp2-mediated p27 degradation. Chem Biol 19: 1515-1524.

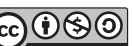

This work is licensed under the Creative Commons Attribution-NonCommercial-Share Alike 3.0 Unported License. To view a copy of this license, visit http://creativecommons. org/licenses/by-nc-sa/3.0/ 